Bull. Korean Math. Soc. 50 (2013), No. 6, pp. 1923-1936

http://dx.doi.org/10.4134/BKMS.2013.50.6.1923

\title{
INTRINSIC SQUARE FUNCTIONS ON FUNCTIONS SPACES INCLUDING WEIGHTED MORREY SPACES
}

\author{
Justin Feuto
}

\begin{abstract}
We prove that the intrinsic square functions including Lusin area integral and Littlewood-Paley $g_{\lambda}^{*}$-function as defined by Wilson, are bounded in a class of function spaces include weighted Morrey spaces. The corresponding commutators generated by $B M O$ functions are also considered.
\end{abstract}

\section{Introduction}

The classical Morrey spaces were introduced by Morrey [13] in connection with partial differential equations. We recall that a real-valued function $f$ is said to belong to the Morrey space $L^{q, \lambda}$ on the $n$-dimensional Euclidean space $\mathbb{R}^{n}$ provided the following norm is finite:

$$
\|f\|_{L^{q, \lambda}}:=\left(\sup _{(y, r) \in \mathbb{R}^{n} \times \mathbb{R}_{+}^{*}} r^{\lambda-n} \int_{B(y, r)}|f(x)|^{q} d x\right)^{\frac{1}{q}} .
$$

Here $1 \leq q<\infty, 0<\lambda<n, \mathbb{R}_{+}^{*}=(0, \infty)$ and $B(y, r)$ is a ball in $\mathbb{R}^{n}$ centered at $y$ of radius $r$.

Chiarenza and Frasca [2] established the boundedness of the Hardy-Littlewood maximal operator, the fractional operator and Calderón-Zygmund operator on these spaces. These operator are also bounded on Lebesgue spaces, and in weighted Lebesgue space $[3,14]$.

Twenty years ago, Fofana introduced a class of function spaces comprising Lebesgue and Morrey spaces [8]. Precisely, for $1 \leq q \leq p \leq \infty$, let $\left(L^{q}, L^{p}\right)\left(\mathbb{R}^{n}\right)$ be the Wiener amalgam space of $L^{q}\left(\mathbb{R}^{n}\right)$ and $L^{p}\left(\mathbb{R}^{n}\right)$, i.e., the space of measurable functions $f: \mathbb{R}^{n} \rightarrow \mathbb{C}$ which are locally in $L^{q}\left(\mathbb{R}^{n}\right)$ and such that the function $y \mapsto\left\|f \chi_{B(y, 1)}\right\|_{q}$ belongs to $L^{p}\left(\mathbb{R}^{n}\right)$, where for $r>0$, $B(y, r)=\left\{x \in \mathbb{R}^{n} /|x-y|<r\right\}$ is the open ball centered at $y$ with radius $r$, $\chi_{B(y, r)}$ its characteristic function and $\|\cdot\|_{q}$ denoting the usual Lebesgue norm in $L^{q}\left(\mathbb{R}^{n}\right)$. As we can see in $[11,9]$, we have the following properties.

Received June 21, 2012; Revised February 23, 2013.

2010 Mathematics Subject Classification. 42B25, 47B38, 47B47.

Key words and phrases. amalgams spaces, Morrey spaces, commutator, $g$-function of Littlewood-Paley, Lusin area function. 
- For $1 \leq q \leq p \leq \infty$, the space $\left(L^{q}, L^{p}\right)\left(\mathbb{R}^{n}\right)$ is a Banach space when it is equipped with the norm

$$
\|f\|_{q, p}:=\left(\int_{\mathbb{R}^{n}}\left\|f \chi_{B(y, 1)}\right\|_{q}^{p}\right)^{\frac{1}{p}}
$$

with the usual modification when $p=\infty$.

- The amalgam space $\left(L^{q}, L^{p}\right)$ is equal to the Lebesgue space $L^{q}$ with equivalence norms, provided $q=p$, while for $q \leq s \leq p$, we have $L^{s}\left(\mathbb{R}^{n}\right)$ continuously embedded in $\left(L^{q}, L^{p}\right)\left(\mathbb{R}^{n}\right)$.

In Lebesgue spaces $L^{q}\left(\mathbb{R}^{n}\right)$, it is well known that for $r>0$ and $x \in \mathbb{R}^{n}$, the dilation operators $\delta_{r}^{q}: f \mapsto r^{\frac{n}{q}} f(r \cdot)$ and the translation operators $\tau_{x}$ : $f \mapsto f(\cdot-x)$ are isometries. We use the usual convention that $\frac{1}{\infty}=0$. When we consider the amalgam spaces $\left(L^{q}, L^{p}\right)\left(\mathbb{R}^{n}\right)$ with $q<p$, only translation operators conserve this property. But it is easy to see that $f \in\left(L^{q}, L^{p}\right)$ if and only if we have

$$
\left\|\delta_{r}^{\alpha} f\right\|_{q, p}<\infty
$$

for all $r>0$ and all $\alpha>0$. Notice that for $1 \leq q, p, \alpha \leq \infty, r>0$ and $\alpha>0$, we have

$$
\begin{aligned}
\left\|\delta_{r}^{\alpha} f\right\|_{q, p} & =r^{n\left(\frac{1}{\alpha}-\frac{1}{q}-\frac{1}{p}\right)}\left(\int_{\mathbb{R}^{n}}\left\|f \chi_{B(y, r)}\right\|_{q}^{p} d y\right)^{\frac{1}{p}} \\
& \approx\left[\int_{\mathbb{R}^{n}}\left(|B(y, r)|^{\frac{1}{\alpha}-\frac{1}{q}-\frac{1}{p}}\left\|f \chi_{B(y, r)}\right\|_{q}\right)^{p} d y\right]^{\frac{1}{p}},
\end{aligned}
$$

where $|B(y, r)|$ stands for the Lebesgue measure of the ball $B(y, r)$. This brings Fofana [8] to consider the subspace $\left(L^{q}, L^{p}\right)^{\alpha}\left(\mathbb{R}^{n}\right)$ of $\left(L^{q}, L^{p}\right)\left(\mathbb{R}^{n}\right)$ that consists in measurable functions $f$ such that $\|f\|_{q, p, \alpha}<\infty$, where for $1 \leq q, p, \alpha \leq \infty$,

$$
\|f\|_{q, p, \alpha}:=\sup _{r>0}\left\|\delta_{r}^{\alpha} f\right\|_{q, p} .
$$

As proved in $[5,7,8]$, the spaces $\left(L^{q}, L^{p}\right)^{\alpha}\left(\mathbb{R}^{n}\right)$ are non trivial if and only if $q \leq \alpha \leq p$. In this case, for fixed $1 \leq q<\alpha$ and $p$ varying from $\alpha$ to $\infty$, these spaces form a chain of distinct Banach spaces beginning with Lebesgue space $L^{\alpha}\left(\mathbb{R}^{n}\right)$ and ending by the classical Morrey space $L^{q, \frac{n q}{\alpha}}\left(\mathbb{R}^{n}\right)=\left(L^{q}, L^{\infty}\right)^{\alpha}\left(\mathbb{R}^{n}\right)$. More precisely, we have the following continuous injections

$$
L^{\alpha}\left(\mathbb{R}^{n}\right) \hookrightarrow\left(L^{q}, L^{p_{1}}\right)^{\alpha}\left(\mathbb{R}^{n}\right) \hookrightarrow\left(L^{q}, L^{p_{2}}\right)^{\alpha}\left(\mathbb{R}^{n}\right) \hookrightarrow L^{q, \frac{n q}{\alpha}}\left(\mathbb{R}^{n}\right)
$$

for $q \leq \alpha<p_{1}<p_{2}<\infty$. It is therefore interesting to know the behavior of operators which are bounded on Lebesgue and Morrey spaces, on these spaces.

We proved in [1] that classical operators such as the Hardy-Littlewood maximal operator, the Calderón-Zygmund operator and Riesz potentials which are

\footnotetext{
${ }^{1}$ Hereafter we propose the following abbreviation $\mathbf{A} \approx \mathbf{B}$ for the inequalities $C^{-1} \mathbf{A} \leq$ $\mathbf{B} \leq C \mathbf{A}$, where $C$ is a positive constant independent of the main parameters.
} 
known to be bounded in Lebesgue and Morrey spaces, are also bounded on $\left(L^{q}, L^{p}\right)^{\alpha}$ spaces (see also $\left.[6]\right)$.

In [15], Komori and Shirai considered the weighted Morrey spaces $L_{w}^{q, \kappa}\left(\mathbb{R}^{n}\right)$ when studying the boundedness of Hardy-Littlewood and Calderón-Zygmund operators.

Let $0<\kappa<1$ and $w$ a weight on $\mathbb{R}^{n}$, i.e., a positive locally integrable function on $\mathbb{R}^{n}$. The weighted Morrey space $L_{w}^{q, \kappa}\left(\mathbb{R}^{n}\right)$, consists of measurable functions $f$ such that $\|f\|_{L_{w}^{q, \kappa}}<\infty$, where

$$
\|f\|_{L_{w}^{q, \kappa}}:=\sup _{B}\left(\frac{1}{w(B)^{\kappa}} \int_{B}|f(x)|^{q} w(x) d x\right)^{\frac{1}{q}} .
$$

These spaces generalize weighted Lebesgue spaces $L_{w}^{q}\left(\mathbb{R}^{n}\right)$, namely the space consisting in measurable functions $f$ satisfying

$$
\|f\|_{q_{w}}:=\left(\int_{\mathbb{R}^{n}}|f(x)|^{q} w(x) d x\right)^{\frac{1}{q}}<\infty .
$$

In this work, we consider for $1 \leq q \leq \alpha \leq p \leq \infty$ and a weight $w$, the space $\left(L_{w}^{q}, L^{p}\right)^{\alpha}\left(\mathbb{R}^{n}\right)$ consists of measurable functions $f$ such that $\|f\|_{q_{w}, p, \alpha}<\infty$, where

$$
r\|f\|_{q_{w}, p, \alpha}:=\left[\int_{\mathbb{R}^{n}}\left(w(B(y, r))^{\frac{1}{\alpha}-\frac{1}{q}-\frac{1}{p}}\left\|f \chi_{B(y, r)}\right\|_{q_{w}}\right)^{p} d y\right]^{\frac{1}{p}}
$$

for $r>0$, and

$$
\|f\|_{q_{w}, p, \alpha}:=\sup _{r>0} r\|f\|_{q_{w}, p, \alpha}
$$

with $w(B(y, r))=\int_{B(y, r)} w(x) d x$ and the usual modification when $p=\infty$. When $w \equiv 1$, we recover $\left(L^{q}, L^{p}\right)^{\alpha}\left(\mathbb{R}^{n}\right)$ spaces while for $q<\alpha$ and $p=\infty$, the spaces $\left(L_{w}^{q}, L^{\infty}\right)^{\alpha}\left(\mathbb{R}^{n}\right)$ are noting but the weighted Morrey spaces $L_{w}^{q, \kappa}\left(\mathbb{R}^{n}\right)$, with $\kappa=\frac{1}{q}-\frac{1}{\alpha}$. Wilson in [18] proved that for $1<q<\infty$ and $0<\gamma \leq 1$, the intrinsic square operators $S_{\gamma}$ given by Relation (2.1), are bounded in the weighted Lebesgue spaces $L_{w}^{q}$, whenever the weight $w$ fulfilled the $\mathcal{A}_{q}$ condition of Muckenhoupt. Wang extends this result to weighted Morrey spaces $L_{w}^{q, \kappa}\left(\mathbb{R}^{n}\right)$. We prove here that these operators and others known to be bounded on weighted Lebesgue and weighted Morrey spaces, are also bounded in the more general setting of $\left(L_{w}^{q}, L^{p}\right)^{\alpha}$ spaces.

This paper is organized as follows:

In Section 2, we recall the definitions of the operators we are going to consider and recall the results on weighted Lebesgue and Morrey spaces. In Section 3 we state our results and in the last section we give their proofs.

Throughout the paper, the letter $C$ is used for non-negative constants that may change from one occurrence to another. The notation $\mathbf{A} \lesssim \mathbf{B}$ will always stand for $\mathbf{A} \leq C \mathbf{B}$, where $C$ is a positive constant independent of the main parameters. For $\alpha>0$ and a ball $B \subset \mathbb{R}^{n}$, we write $\alpha B$ for the ball with same center as $B$ and with radius $\alpha$ times radius of $B$. For any subset $E$ of $\mathbb{R}^{n}$, we 
denote $E^{c}:=\mathbb{R}^{n} \backslash E$ the complement of $E$. We denote by $\mathbb{N}^{*}$ the set of all positive integers.

\section{Definitions and known results}

For $0<\gamma \leq 1$, we denote by $\mathcal{C}_{\gamma}$ the family of function $\varphi$ defined on $\mathbb{R}^{n}$ with support in the closed unit ball $\mathbb{B}=\left\{x \in \mathbb{R}^{n}:|x| \leq 1\right\}$ and vanishing integral, i.e., $\int_{\mathbb{R}^{n}} \varphi(x) d x=0$, and such that for all $x, x^{\prime} \in \mathbb{R}^{n},\left|\varphi(x)-\varphi\left(x^{\prime}\right)\right| \leq\left|x-x^{\prime}\right|^{\gamma}$. Let $\mathbb{R}_{+}^{n+1}=\mathbb{R}^{n} \times(0, \infty)$ and $\varphi_{t}(x)=t^{-n} \varphi\left(t^{-1} x\right)$. The intrinsic square function of $f$ (of order $\gamma$ ) is defined by the formula

$$
S_{\gamma}(f)(x)=\left[\int_{\Gamma(x)}\left(\sup _{\varphi \in \mathcal{C}_{\gamma}}\left|f * \varphi_{t}(y)\right|\right)^{2} \frac{d y d t}{t^{n+1}}\right]^{\frac{1}{2}},
$$

where for $x \in \mathbb{R}^{n}, \Gamma(x)$ denote the usual "cone of arperture one",

$$
\Gamma(x)=\left\{(y, t) \in \mathbb{R}_{+}^{n+1}:|x-y|<t\right\} .
$$

For $1<q<\infty$ and $0<\gamma \leq 1$, the operators $S_{\gamma}$ are bounded on $L_{w}^{q}\left(\mathbb{R}^{n}\right)$ provided $w \in \mathcal{A}_{q}$ [17]. We recall that a weight $w$ is of class $\mathcal{A}_{q}$ or belongs to $\mathcal{A}_{q}$ for $1<q<\infty$ if there exists a constant $C>0$ such that for all balls $B \subset \mathbb{R}^{n}$ we have

$$
\left(\frac{1}{|B|} \int_{B} w(x) d x\right)\left(\frac{1}{|B|} \int_{B} w^{\frac{-1}{q-1}}(x) d x\right)^{q-1} \leq C .
$$

In the setting of weighted Morrey spaces one has the following.

Theorem 2.1 (Theorem $1.1[16]$ ). Let $0<\gamma \leq 1,1<q<\infty, 0<\kappa<1$ and $w \in \mathcal{A}_{q}$. Then there exists $C>0$ such that

$$
\left\|S_{\gamma} f\right\|_{L_{w}^{q, \kappa}} \leq C\|f\|_{L_{w}^{q, \kappa}} .
$$

We also define the intrinsic Littlewood-Paley $g$-function $g_{\gamma}(f)$ and $g_{\lambda}^{*}$-function $g_{\lambda, \gamma}^{*}(f)$ by

$$
g_{\gamma}(f)(x)=\left[\int_{0}^{\infty}\left(\sup _{\varphi \in \mathcal{C}_{\gamma}}\left|f * \varphi_{t}(y)\right|\right)^{2} \frac{d t}{t}\right]^{\frac{1}{2}}
$$

and

$$
g_{\lambda, \gamma}^{*}(f)(x)=\left[\int_{\mathbb{R}_{+}^{n+1}}\left(\frac{t}{t+|x-y|}\right)^{\lambda n}\left(\sup _{\varphi \in \mathcal{C}_{\gamma}}\left|f * \varphi_{t}(y)\right|\right)^{2} \frac{d y d t}{t^{n+1}}\right]^{\frac{1}{2}}
$$

respectively.

Theorem 2.2 (Theorem $1.3[16]$ ). Let $0<\gamma \leq 1,1<q<\infty, 0<\kappa<1$ and $w \in \mathcal{A}_{q}$. If $\lambda>\max \{q, 3\}$, then there exists $C>0$ such that

$$
\left\|g_{\lambda, \gamma}^{*} f\right\|_{L_{w}^{q, \kappa}} \leq C\|f\|_{L_{w}^{q, \kappa}} .
$$


Let $b$ be a locally integrable function. The commutator of $b$ and $S_{\gamma}$ is defined by

$$
\left[b, S_{\gamma}\right](f)(x)=\left(\int_{\Gamma(x)} \sup _{\varphi \in \mathcal{C}_{\gamma}}\left|\int_{\mathbb{R}^{n}}(b(x)-b(z)) \varphi_{t}(y-z) f(z) d z\right|^{2} \frac{d y d t}{t^{n+1}}\right)^{\frac{1}{2}},
$$

and the commutator of $b$ and $g_{\lambda, \gamma}^{*}$ by

$$
\begin{aligned}
& {\left[b, g_{\lambda, \gamma}^{*}\right](f)(x) } \\
= & \left(\int_{\mathbb{R}_{+}^{n+1}}\left(\frac{t}{t+|x-y|}\right)^{\lambda n} \sup _{\varphi \in \mathcal{C}_{\gamma}}\left|\int_{\mathbb{R}^{n}}(b(x)-b(z)) \varphi_{t}(y-z) f(z) d z\right|^{2} \frac{d y d t}{t^{n+1}}\right)^{\frac{1}{2}} .
\end{aligned}
$$

A locally integrable function $b$ belongs to $B M O\left(\mathbb{R}^{n}\right)$ (bounded mean oscillation functions) if $\|b\|_{B M O\left(\mathbb{R}^{n}\right)}<\infty$, where

$$
\|b\|_{B M O\left(\mathbb{R}^{n}\right)}:=\sup _{B: \text { ball }} \frac{1}{|B|} \int_{B}\left|b(x)-b_{B}\right| d x .
$$

We have the following result in the context of weighted Lebesgue spaces.

Theorem 2.3 (Theorem $3.1[16]$ ). Let $0<\gamma \leq 1,1<q<\infty$ and $w \in \mathcal{A}_{q}$. Then the commutators $\left[b, S_{\gamma}\right]$ and $\left[b, g_{\lambda, \gamma}^{*}\right]$ are bounded on $L_{w}^{q}\left(\mathbb{R}^{n}\right)$ whenever $b \in B M O\left(\mathbb{R}^{n}\right)$.

For weighted Morrey spaces, the following results are proved.

Theorem 2.4 (Theorem $1.2[16]$ ). Let $0<\gamma \leq 1,1<q<\infty, 0<\kappa<1$ and $w \in \mathcal{A}_{q}$. Suppose that $b \in B M O$, then there exists $C>0$ such that

$$
\left\|\left[b, S_{\gamma}\right] f\right\|_{L_{w}^{q, \kappa}} \leq C\|f\|_{L_{w}^{q, \kappa}} .
$$

Theorem 2.5 (Theorem $1.4[16]$ ). Let $0<\gamma \leq 1,1<q<\infty, 0<\kappa<1$ and $w \in \mathcal{A}_{q}$. If $b \in B M O\left(\mathbb{R}^{n}\right)$ and $\lambda>\max \{q, 3\}$, then there is a constant $C>0$ independent of $f$ such that

$$
\left\|\left[b, g_{\lambda, \gamma}^{*}\right](f)\right\|_{L_{w}^{q, \kappa}} \leq C\|f\|_{L_{w}^{q, \kappa}} .
$$

\section{Statement of our main results}

Since our space at least for the case where the weight is equal to 1 , is included in Morrey spaces, we already know that the image is in the space of Morrey. But what is shown is that if one has a slightly stronger assumption, then this is also true for the image.

For the intrinsic square function $S_{\gamma}$, we have the following result.

Theorem 3.1. Let $0<\gamma \leq 1,1<q \leq \alpha<p \leq \infty$ and $w \in \mathcal{A}_{q}$. The operators $S_{\gamma}$ are bounded in $\left(L_{w}^{q}, L^{p}\right)^{\alpha}\left(\mathbb{R}^{n}\right)$.

Theorem 2.1 is a particular case of this result. The next, concerning the intrinsic Littlewood-Paley $g_{\lambda}^{*}$-function is an extension of Theorem 2.2. 
Theorem 3.2. Let $0<\gamma \leq 1,1<q \leq \alpha<p \leq \infty$ and $w \in \mathcal{A}_{q}$. If $\lambda>\max \{q, 3\}$, then there exists a constant $C>0$ such that

$$
\left\|g_{\lambda, \gamma}^{*}(f)\right\|_{q_{w}, p, \alpha} \leq C\|f\|_{q_{w}, p, \alpha}
$$

for all $f \in\left(L_{w}^{q}, L^{p}\right)^{\alpha}\left(\mathbb{R}^{n}\right)$.

Furthermore, we have the following results which are extensions of Theorems 2.4 and 2.5 , respectively.

Theorem 3.3. Let $0<\gamma \leq 1,1<q \leq \alpha<p \leq \infty$ and $w \in \mathcal{A}_{q}$. Suppose that $b \in B M O\left(\mathbb{R}^{n}\right)$, then there exists a constant $C>0$ not depending on $f$ such that

for all $f \in\left(L_{w}^{q}, L^{p}\right)^{\alpha}\left(\mathbb{R}^{n}\right)$.

$$
\left\|\left[b, S_{\gamma}\right](f)\right\|_{q_{w}, p, \alpha} \leq C\|f\|_{q_{w}, p, \alpha}
$$

Theorem 3.4. Let $0<\gamma \leq 1,1<q \leq \alpha<p \leq \infty$ and $w \in \mathcal{A}_{q}$. If $b \in B M O\left(\mathbb{R}^{n}\right)$ and $\lambda>\max \{q, 3\}$, then there exists a constant $C>0$ such that

$$
\left\|\left[b, g_{\lambda, \gamma}^{*}\right](f)\right\|_{q_{w}, p, \alpha} \leq C\|f\|_{q_{w}, p, \alpha}
$$

for all $f \in\left(L_{w}^{q}, L^{p}\right)^{\alpha}\left(\mathbb{R}^{n}\right)$.

Since for any $0<\gamma \leq 1$ the functions $S_{\gamma}(f)$ and $g_{\gamma}(f)$ are pointwise comparable (see [17]), as an immediate consequence of Theorems 3.1 and 3.3 we have the following results.

Corollary 3.5. Let $0<\gamma \leq 1,1<q \leq \alpha<p \leq \infty$ and $w \in \mathcal{A}_{q}$. The operator $g_{\gamma}$ is bounded in $\left(L_{w}^{q}, L^{p}\right)^{\alpha}\left(\mathbb{R}^{n}\right)$.

Corollary 3.6. Let $0<\gamma \leq 1,1<q \leq \alpha<p \leq \infty$ and $w \in \mathcal{A}_{q}$. Suppose that $b \in B M O\left(\mathbb{R}^{n}\right)$, then there exists a constant $C>0$ not depending on $f$ such that

$$
\left\|\left[b, g_{\gamma}\right](f)\right\|_{q_{w}, p, \alpha} \leq C\|f\|_{q_{w}, p, \alpha}
$$

for all $f \in\left(L_{w}^{q}, L^{p}\right)^{\alpha}\left(\mathbb{R}^{n}\right)$.

The above corollaries are extensions of Corollary 1.5 and Corollary 1.6 of [16], respectively.

\section{Proof of the main results}

We will need the following properties of $\mathcal{A}_{q}$ weights (see Proposition 9.1.5 and Theorem 9.2.2[10]). Let $w \in \mathcal{A}_{q}$ for some $1<q<\infty$.

(1) For all $\lambda>1$ and all balls $B$, we have

$$
w(\lambda B) \lesssim \lambda^{n q} w(B) .
$$


(2) There exists a positive constant $\tau$ such that for every ball $B$, we have

$$
\left(\frac{1}{|B|} \int_{B} w(t)^{1+\tau} d t\right)^{\frac{1}{1+\tau}} \lesssim \frac{1}{|B|} \int_{B} w(t) d t
$$

and for any measurable subset $E$ of a ball $B$, we have

$$
\frac{w(E)}{w(B)} \lesssim\left(\frac{|E|}{|B|}\right)^{\frac{\tau}{1+\tau}}
$$

For our proofs, we use arguments as in [4].

Proof of Theorem 3.1. We fix $r>0$ and let $B=B(y, r)$ for some $y \in \mathbb{R}^{n}$. We write $f=f_{1}+f_{2}$, with $f_{1}=f \chi_{2 B}$. Since $S_{\gamma}$ is a sublinear operator, we have

$$
\left\|S_{\gamma}(f) \chi_{B}\right\|_{q_{w}} \leq\left\|S_{\gamma}\left(f_{1}\right) \chi_{B}\right\|_{q_{w}}+\left\|S_{\gamma}\left(f_{2}\right) \chi_{B}\right\|_{q_{w}} .
$$

For the term in $f_{1}$, we have

$$
\left\|S_{\gamma}\left(f_{1}\right) \chi_{B}\right\|_{q_{w}} \lesssim\left\|f \chi_{2 B}\right\|_{q_{w}}
$$

as an immediate consequence of the boundedness of $S_{\gamma}$ in $L_{w}^{q}\left(\mathbb{R}^{n}\right)$. Our attention will be focused now on the second term.

Let $\varphi \in \mathcal{C}_{\gamma}$, and $t>0$. Since the family $\mathcal{C}_{\gamma}$ is uniformly bounded with respect to the $L^{\infty}$-norm, we have

$$
\left|f_{2} * \varphi_{t}(u)\right| \lesssim t^{-n} \int_{(2 B)^{c} \cap \tilde{B}(u, t)}|f(z)| d z
$$

for all $u \in \mathbb{R}^{n}$, where $\tilde{B}(u, t):=\left\{z \in \mathbb{R}^{n} /|z-u| \leq t\right\}$. Thus for all $x \in \mathbb{R}^{n}$, we have

$$
\begin{aligned}
\left|S_{\gamma}\left(f_{2}\right)(x)\right| & \lesssim\left[\int_{\Gamma(x)}\left(t^{-n} \int_{(2 B)^{c} \cap \tilde{B}(u, t)}|f(z)| d z\right)^{2} \frac{d u d t}{t^{n+1}}\right]^{\frac{1}{2}} \\
& \lesssim \sum_{k=1}^{\infty} \int_{2^{k+1} B \backslash 2^{k} B}|f(z)|\left[\int_{0}^{\infty}\left(\int_{B(x, t)} \chi_{\tilde{B}(z, t)}(u) d u\right) \frac{d t}{t^{3 n+1}}\right]^{\frac{1}{2}} d z
\end{aligned}
$$

where the last control is an application of Minkowski's integral inequality.

We suppose $x \in B(y, r)$. For $k \in \mathbb{N}^{*}, z \in 2^{k+1} B \backslash 2^{k} B$ and $t>0$, $\int_{B(x, t)} \chi_{\tilde{B}(z, t)}(u) d u \neq 0$ implies that $B(x, t) \cap \tilde{B}(z, t) \neq \emptyset$. Let $u_{0} \in B(x, t) \cap$ $\tilde{B}(z, t)$, we have

$$
2 t \geq\left|x-u_{0}\right|+\left|z-u_{0}\right| \geq|x-z| \geq|y-z|-|x-y| \geq 2^{k-1} r .
$$

Thus for $x \in B=B(y, r)$,

$$
\left|S_{\gamma}\left(f_{2}\right)(x)\right| \lesssim \sum_{k=1}^{\infty} \int_{2^{k+1} B \backslash 2^{k} B}|f(z)|\left(\int_{2^{k-2} r}^{\infty} \int_{B(x, t)} d u \frac{d t}{t^{3 n+1}}\right)^{\frac{1}{2}} d z
$$




$$
\begin{aligned}
& \lesssim \sum_{k=1}^{\infty} \int_{2^{k+1} B \backslash 2^{k} B}|f(z)|\left(\int_{2^{k-2} r}^{\infty} \frac{d t}{t^{2 n+1}}\right)^{\frac{1}{2}} d z \\
& \lesssim \sum_{k=1}^{\infty} \frac{1}{\left|2^{k+1} B\right|} \int_{2^{k+1} B \backslash 2^{k} B}|f(z)| d z .
\end{aligned}
$$

However, by Hölder Inequality and (2.2), we have for every $k \in \mathbb{N}^{*}$

$$
\frac{1}{\left|2^{k+1} B\right|} \int_{2^{k+1} B}|f(z)| d z \lesssim\left\|f \chi_{2^{k+1} B}\right\|_{q_{w}} w\left(2^{k+1} B\right)^{-\frac{1}{q}} .
$$

It follows that

$$
\left\|S_{\gamma}\left(f_{2}\right) \chi_{B(y, r)}\right\|_{q_{w}} \lesssim \sum_{k=1}^{\infty}\left\|f \chi_{2^{k+1} B}\right\|_{q_{w}}\left(\frac{w(B)}{w\left(2^{k+1} B\right)}\right)^{\frac{1}{q}}
$$

Multiplying both inequalities (4.5) and (4.9) by $w(B(y, r))^{\frac{1}{\alpha}-\frac{1}{q}-\frac{1}{p}}$, it comes from (4.3) that

$$
\begin{aligned}
& w(B(y, r))^{\frac{1}{\alpha}-\frac{1}{q}-\frac{1}{p}}\left\|S_{\gamma}(f) \chi_{B(y, r)}\right\|_{q_{w}} \\
\lesssim & w(B(y, 2 r))^{\frac{1}{\alpha}-\frac{1}{q}-\frac{1}{p}}\left\|f \chi_{B(y, 2 r)}\right\|_{q_{w}} \\
& +\sum_{k=1}^{\infty} w\left(B\left(y, 2^{k+1} r\right)\right)^{\frac{1}{\alpha}-\frac{1}{q}-\frac{1}{p}}\left\|f \chi_{B\left(y, 2^{k+1} r\right)}\right\|_{q_{w}} \frac{1}{2^{\frac{n k}{s}\left(\frac{1}{\alpha}-\frac{1}{p}\right)}}
\end{aligned}
$$

for some $s>0$. Therefore the $L^{p}$ norm of both sides of (4.10) leads to

$$
r\left\|S_{\gamma}(f)\right\|_{q_{w}, p, \alpha} \lesssim\left(1+\sum_{k=1}^{\infty} \frac{1}{2^{\frac{n k}{s}\left(\frac{1}{\alpha}-\frac{1}{p}\right)}}\right)\|f\|_{q_{w}, p, \alpha}, r>0,
$$

and the result follows, since the series on the right hand side converge.

For the proof of Theorem 3.2, we need the following varying-aperture versions of $S_{\gamma}$. For $0<\gamma \leq 1$ and $\beta>0$, we define $S_{\gamma, \beta}(f)$ by

$$
S_{\gamma, \beta}(f)(x)=\left[\int_{\Gamma_{\beta}(x)}\left(\sup _{\varphi \in \mathcal{C}_{\gamma}}\left|f * \varphi_{t}(y)\right|\right)^{2} \frac{d y d t}{t^{n+1}}\right]^{\frac{1}{2}},
$$

where $\Gamma_{\beta}(x)=\left\{(x, t) \in \mathbb{R}_{+}^{n+1} /|x-y|<\beta t\right\}$. We have the following lemma which is a consequence of Lemmas 1.1, 1.2 and 1.3 of [16] and the boundedness of $S_{\gamma}:=S_{\gamma, 2^{0}}$ on the weighted Lebesgue spaces.

Lemma 4.1. Let $0<\gamma \leq 1,1<q<\infty$ and $w \in \mathcal{A}_{q}$. Then for all non negative integers $j, S_{\gamma, 2^{j}}$ is bounded on $L_{w}^{q}\left(\mathbb{R}^{n}\right)$. Moreover

$$
\left\|S_{\gamma, 2^{j}}(f)\right\|_{q_{w}} \lesssim\left(2^{n j}+2^{\frac{n j q}{2}}\right)\|f\|_{q_{w}} .
$$


Proof of Theorem 3.2. For all $x \in \mathbb{R}^{n}$, we have

$$
g_{\lambda, \gamma}^{*}(f)(x)^{2} \lesssim S_{\gamma}(f)(x)^{2}+\sum_{j=1}^{\infty} 2^{-j \lambda n} S_{\gamma, 2^{j}}(f)(x)^{2} .
$$

Fix $r>0$. For $y \in \mathbb{R}^{n}$ and $B=B(y, r)$ a ball in $\mathbb{R}^{n}$, we have

$$
\begin{aligned}
& w(B)^{\frac{1}{\alpha}-\frac{1}{q}-\frac{1}{p}}\left\|g_{\lambda, \gamma}^{*}(f) \chi_{B}\right\|_{q_{w}} \\
\lesssim & w(B)^{\frac{1}{\alpha}-\frac{1}{q}-\frac{1}{p}}\left\|S_{\gamma}(f) \chi_{B}\right\|_{q_{w}} \\
& +\sum_{j=1}^{\infty} 2^{-j \lambda n / 2} w(B)^{\frac{1}{\alpha}-\frac{1}{q}-\frac{1}{p}}\left\|S_{\gamma, 2^{j}}(f) \chi_{B}\right\|_{q_{w}},
\end{aligned}
$$

according to (4.13). By Theorem 3.1, we have that the $L^{p}$ norm of the first term on the right hand side of (4.14) is controlled by $\|f\|_{q_{w}, p, \alpha}$. Let $j$ be fixed in $\mathbb{N}^{*}$. For $S_{\gamma, 2^{j}} f$, we proceed as for $S_{\gamma} f$. So, for $f=f_{1}+f_{2}$ with $f_{1}=f \chi_{2 B}$, we have

$$
\begin{aligned}
w(B)^{\frac{1}{\alpha}-\frac{1}{q}-\frac{1}{p}}\left\|S_{\gamma, 2^{j}}(f) \chi_{B}\right\|_{q_{w}} \leq & w(B)^{\frac{1}{\alpha}-\frac{1}{q}-\frac{1}{p}}\left\|S_{\gamma, 2^{j}}\left(f_{1}\right) \chi_{B}\right\|_{q_{w}} \\
& +w(B)^{\frac{1}{\alpha}-\frac{1}{q}-\frac{1}{p}}\left\|S_{\gamma, 2^{j}}\left(f_{2}\right) \chi_{B}\right\|_{q_{w}} .
\end{aligned}
$$

Applying Lemma 4.1 and taking into consideration (4.1), we obtain (4.16)

$$
w(B)^{\frac{1}{\alpha}-\frac{1}{q}-\frac{1}{p}}\left\|S_{\gamma, 2^{j}}\left(f_{1}\right) \chi_{B}\right\|_{q_{w}} \lesssim\left(2^{j n}+2^{j n q / 2}\right) w(2 B)^{\frac{1}{\alpha}-\frac{1}{q}-\frac{1}{p}}\left\|f \chi_{2 B}\right\|_{q_{w}} .
$$

Let us estimate now the term in $f_{2}$. The same arguments we use to estimate $S_{\gamma}\left(f_{2}\right)(x)$ for $x \in B$, i.e., Minkowsky's integral inequality and the fact that for $k \in \mathbb{N}^{*}, z \in 2^{k+1} B \backslash 2^{k} B$

$$
\int_{B\left(x, 2^{j} t\right)} \chi_{\tilde{B}(z, t)}(u) d u \neq 0 \Rightarrow t \geq \frac{2^{k-1}}{2^{j}+1} r,
$$

allow us to get the following

$$
\begin{aligned}
\left|S_{\gamma, 2^{j}}\left(f_{2}\right)(x)\right| & \lesssim 2^{3 j n / 2} \sum_{k=1}^{\infty} \frac{1}{\left|2^{k+1} B\right|} \int_{2^{k+1} B \backslash 2^{k} B}|f(z)| d z \\
& \lesssim 2^{3 j n / 2} \sum_{k=1}^{\infty}\left\|f \chi_{B\left(y, 2^{k+1} r\right)}\right\|_{q_{w}} w\left(B\left(y, 2^{k+1} r\right)\right)^{-\frac{1}{q}}
\end{aligned}
$$

for all $x \in B(y, r)$, where the last control comes from Estimation (4.8). Therefore, its $L_{w}^{q}(B)$-norm leads to

$$
w(B)^{\frac{1}{\alpha}-\frac{1}{q}-\frac{1}{p}}\left\|S_{\gamma, 2^{j}}\left(f_{2}\right) \chi_{B}\right\|_{q_{w}} \lesssim 2^{3 j n / 2} \sum_{k=1}^{\infty} \frac{w\left(2^{k+1} B\right)^{\frac{1}{\alpha}-\frac{1}{q}-\frac{1}{p}}}{2^{\frac{n k}{s}\left(\frac{1}{\alpha}-\frac{1}{p}\right)}}\left\|f \chi_{2^{k+1} B}\right\|_{q_{w}} .
$$


Taking estimates (4.16) and (4.17) in (4.15), we have

$$
\begin{aligned}
& w(B(y, r))^{\frac{1}{\alpha}-\frac{1}{q}-\frac{1}{p}}\left\|S_{\gamma, 2^{j}}(f) \chi_{B(y, r)}\right\|_{q_{w}} \\
\lesssim & \left(2^{j n}+2^{j n q / 2}\right) w(B(y, 2 r))^{\frac{1}{\alpha}-\frac{1}{q}-\frac{1}{p}}\left\|f \chi_{B(y, 2 r)}\right\|_{q_{w}} \\
& +2^{3 j n / 2} \sum_{k=1}^{\infty} \frac{w\left(B\left(y, 2^{k+1} r\right)\right)^{\frac{1}{\alpha}-\frac{1}{q}-\frac{1}{p}}}{2^{\frac{n k}{s}\left(\frac{1}{\alpha}-\frac{1}{p}\right)}}\left\|f \chi_{B\left(y, 2^{k+1} r\right)}\right\|_{q_{w}}
\end{aligned}
$$

for all $y \in \mathbb{R}^{n}$, so that the $L^{p}$-norm of both sides leads to

$$
r\left\|S_{\gamma, 2^{j}}(f)\right\|_{q_{w}, p, \alpha} \lesssim\left(2^{j n}+2^{j n q / 2}\right)\|f\|_{q_{w}, p, \alpha}+\|f\|_{q_{w}, p, \alpha} 2^{3 j n / 2} .
$$

Therefore the $L^{p}$ norm of (4.14) give

$$
\begin{aligned}
r\left\|g_{\lambda, \gamma}^{*}(f)\right\|_{q_{w}, p, \alpha} & \lesssim\left(1+\sum_{j=1}^{\infty} 2^{-j \lambda n / 2}\left(2^{j n}+2^{j n q / 2}+2^{3 j n / 2}\right)\right)\|f\|_{q_{w}, p, \alpha} \\
& \lesssim\|f\|_{q_{w}, p, \alpha}
\end{aligned}
$$

for $r>0$, where the convergence of the series is due to the fact that $\lambda>$ $\max \{q, 3\}$. We end the proof by taking the supremum over all $r>0$.

For the proof of the next results on commutators, we use the following properties of $B M O$ (see [12]). Let $b$ be a locally integrable function. If $b \in$ $B M O\left(\mathbb{R}^{n}\right)$, then for every $1<p<\infty$, we have

$$
\|b\|_{B M O\left(\mathbb{R}^{n}\right)} \approx \sup _{B: \text { ball }}\left(\frac{1}{|B|} \int_{B}\left|b(x)-b_{B}\right|^{p} d x\right)^{\frac{1}{p}},
$$

and for $w \in \mathcal{A}_{q}$ with $1<q<\infty$,

$$
\left(\frac{1}{w(B)} \int_{B}\left|b(x)-b_{B}\right|^{p} w(x) d x\right)^{\frac{1}{p}} \lesssim\|b\|_{B M O},
$$

which is an immediate consequence of (4.20) and the characterization (4.2) of $\mathcal{A}_{q}$ weights.

Proof of Theorem 3.3. Fix $y \in \mathbb{R}^{n}$ and $r>0$. For $B=B(y, r)$, we put $f=$ $f_{1}+f_{2}$ with $f_{1}=f \chi_{2 B}$. We have

$$
\begin{aligned}
w(B)^{\frac{1}{\alpha}-\frac{1}{q}-\frac{1}{p}}\left\|\left[b, S_{\gamma}\right](f) \chi_{B}\right\|_{q_{w}} \leq & w(B)^{\frac{1}{\alpha}-\frac{1}{q}-\frac{1}{p}}\left\|\left[b, S_{\gamma}\right]\left(f_{1}\right) \chi_{B}\right\|_{q_{w}} \\
& +w(B)^{\frac{1}{\alpha}-\frac{1}{q}-\frac{1}{p}}\left\|\left[b, S_{\gamma}\right]\left(f_{2}\right) \chi_{B}\right\|_{q_{w}} .
\end{aligned}
$$

For the term in $f_{1}$, it is immediate that

$$
w(B(y, r))^{\frac{1}{\alpha}-\frac{1}{q}-\frac{1}{p}}\left\|\left[b, S_{\gamma}\right]\left(f_{1}\right) \chi_{B(y, r)}\right\|_{q_{w}} \lesssim w(B(y, 2 r))^{\frac{1}{\alpha}-\frac{1}{q}-\frac{1}{p}}\left\|f \chi_{B(y, 2 r)}\right\|_{q_{w}},
$$

according to the boundedness of the commutator on $L_{w}^{q}\left(\mathbb{R}^{n}\right)$ and (4.1). It remains to estimate the term in $f_{2}$. 
Let $x \in \mathbb{R}^{n}$. For $(u, t) \in \Gamma(x)$, we have

$$
\begin{aligned}
& \sup _{\varphi \in \mathcal{C}_{\gamma}}\left|\int_{\mathbb{R}^{n}}(b(x)-b(z)) \varphi_{t}(u-z) f_{2}(z) d z\right| \\
\leq & \left|b(x)-b_{B}\right| \sup _{\varphi \in \mathcal{C}_{\gamma}}\left|f_{2} * \varphi_{t}(u)\right|+\sup _{\varphi \in \mathcal{C}_{\gamma}}\left|\left(b-b_{B}\right) f_{2} * \varphi_{t}(u)\right|
\end{aligned}
$$

so that the $L^{2}\left(\Gamma(x), \frac{d u d t}{t^{n+1}}\right)$-norm of both sides leads to

$$
\begin{aligned}
\left|\left[b, S_{\gamma}\right] f_{2}(x)\right| \leq & \left|b(x)-b_{B}\right| S_{\gamma}\left(f_{2}\right)(x) \\
& +\left\{\int_{\Gamma(x)}\left(\sup _{\varphi \in \mathcal{C}_{\gamma}}\left|\left[\left(b-b_{B}\right) f_{2}\right] * \varphi_{t}(u)\right|\right)^{2} \frac{d u d t}{t^{n+1}}\right\}^{\frac{1}{2}} \\
= & I+I I .
\end{aligned}
$$

We take $x \in B=B(y, r)$. As we proved in Theorem 3.1, we have

$$
\left|S_{\gamma}\left(f_{2}\right)(x)\right| \lesssim \sum_{k=1}^{\infty}\left\|f \chi_{2^{k+1} B}\right\|_{q_{w}} w\left(2^{k+1} B\right)^{-\frac{1}{q}}
$$

Thus, the $L_{w}^{q}(B)$-norm of $I$ can be estimated as follow

(4.24) $\left\|\left|b-b_{B}\right| S_{\gamma}\left(f_{2}\right) \chi_{B}\right\|_{q_{w}} \lesssim\|b\|_{B M O} \sum_{k=1}^{\infty}\left(\frac{w(B)}{w\left(2^{k+1} B\right)}\right)^{\frac{1}{q}}\left\|f \chi_{2^{k+1} B}\right\|_{q_{w}}$,

where we use (4.21). On the other hand, it comes from the uniform boundedness of the family $\mathcal{C}_{\gamma}$ that

$$
I I \lesssim\left[\int_{\Gamma(x)}\left(t^{-n} \int_{(2 B)^{c} \cap \tilde{B}(u, t)}\left|b(z)-b_{B}\right||f(z)| d z\right)^{2} \frac{d u d t}{t^{n+1}}\right]^{\frac{1}{2}},
$$

so that using once more Minkowski's inequality for integrals and inequality (4.7), we have

$$
\begin{aligned}
I I \lesssim & \sum_{k=1}^{\infty} \frac{1}{\left|2^{k+1} B\right|} \int_{2^{k+1} B \backslash 2^{k} B}\left|b(z)-b_{B}\right||f(z)| d z \\
\leq & \sum_{k=1}^{\infty} \frac{1}{\left|2^{k+1} B\right|} \int_{2^{k+1} B \backslash 2^{k} B}\left|b(z)-b_{2^{k+1} B}\right||f(z)| d z \\
& +\sum_{k=1}^{\infty} \frac{\left|b_{2^{k+1} B}-b_{B}\right|}{\left|2^{k+1} B\right|} \int_{2^{k+1} B \backslash 2^{k} B}|f(z)| d z
\end{aligned}
$$

for all $x \in B(y, r)$. However,

$$
\int_{\left(2^{k+1} B \backslash 2^{k} B\right)}\left|b(z)-b_{2^{k+1} B}\right||f(z)| d z
$$




$$
\begin{aligned}
& \leq\left(\int_{2^{k+1} B}\left|b(z)-b_{2^{k+1} B}\right|^{q^{\prime}} w(z)^{-\frac{q^{\prime}}{q}} d z\right)^{\frac{1}{q^{\prime}}}\left(\int_{2^{k+1} B}|f(z)|^{q} w(z) d z\right)^{\frac{1}{q}} \\
& \lesssim\left\|f \chi_{2^{k+1} B}\right\|_{q_{w}}\left|2^{k+1} B\right| w\left(2^{k+1} B\right)^{-\frac{1}{q}}\|b\|_{B M O},
\end{aligned}
$$

according to Hölder inequality and the fact that the weight $v(z)=w(z)^{-\frac{q^{\prime}}{q}}$ belongs to $\mathcal{A}_{q^{\prime}}$ whenever $w \in \mathcal{A}_{q}$. So

$$
\begin{aligned}
\sigma_{1} & :=\sum_{k=1}^{\infty} \frac{1}{\left|2^{k+1} B\right|} \int_{2^{k+1} B \backslash 2^{k} B}\left|b(z)-b_{2^{k+1} B}\right||f(z)| d z \\
& \lesssim\|b\|_{B M O} \sum_{k=1}^{\infty}\left\|f \chi_{2^{k+1} B}\right\|_{q_{w}} w\left(2^{k+1} B\right)^{-\frac{1}{q}}
\end{aligned}
$$

on $B$, and the $L_{w}^{q}(B)$ norm of both sides leads to

$$
\left\|\sigma_{1} \chi_{B(y, r)}\right\|_{q_{w}} \lesssim\|b\|_{B M O} \sum_{k=1}^{\infty}\left\|f \chi_{2^{k+1} B}\right\|_{q_{w}}\left(\frac{w(B)}{w\left(2^{k+1} B\right)}\right)^{\frac{1}{q}} .
$$

For the second series, we have

$$
\begin{aligned}
\sigma_{2} & :=\sum_{k=1}^{\infty} \frac{\left|b_{2^{k+1} B}-b_{B}\right|}{\left|2^{k+1} B\right|} \int_{2^{k+1} B \backslash 2^{k} B}|f(z)| d z \\
& \lesssim\|b\|_{B M O}\left(\sum_{k=1}^{\infty}(k+1)\left\|f \chi_{2^{k+1} B}\right\|_{q_{w}} w\left(2^{k+1} B\right)^{-\frac{1}{q}}\right),
\end{aligned}
$$

where we use the fact that $\left|b_{2^{k+1} B}-b_{B}\right| \lesssim(k+1)\|b\|_{B M O}$ and Relation (4.8). It comes that

$$
\left\|\sigma_{2} \chi_{B(y, r)}\right\|_{q_{w}} \lesssim\|b\|_{B M O}\left(\sum_{k=1}^{\infty}(k+1)\left\|f \chi_{2^{k+1} B}\right\|_{q_{w}}\left(\frac{w(B)}{w\left(2^{k+1} B\right)}\right)^{\frac{1}{q}}\right) .
$$

Hence, putting together (4.24), (4.25) and (4.26), we obtain,

$$
\begin{aligned}
& w(B(y, r))^{\frac{1}{\alpha}-\frac{1}{q}-\frac{1}{p}}\left\|\left[b, S_{\eta}\right]\left(f_{2}\right) \chi_{B(y, r)}\right\|_{q_{w}} \\
\lesssim & \|b\|_{B M O}\left(\sum_{k=1}^{\infty} \frac{k+3}{2^{\frac{2 n k}{s^{\prime}}\left(\frac{1}{\alpha}-\frac{1}{p}\right)}} w\left(B\left(y, 2^{k+1} r\right)\right)^{\frac{1}{\alpha}-\frac{1}{q}-\frac{1}{p}}\left\|f \chi_{B\left(y, 2^{k+1} r\right)}\right\|_{q_{w}}\right)
\end{aligned}
$$

for all $y \in \mathbb{R}^{n}$ and some $s^{\prime}>0$. Taking estimates (4.23) and (4.27) in (4.22) yield,

$$
\begin{aligned}
& w(B(y, r))^{\frac{1}{\alpha}-\frac{1}{q}-\frac{1}{p}}\left\|\left[b, S_{\eta}\right](f) \chi_{B(y, r)}\right\|_{q_{w}} \\
\lesssim & \|b\|_{B M O}\left(\sum_{k=1}^{\infty} \frac{k+3}{2^{\frac{2 n k}{s^{\prime}}\left(\frac{1}{\alpha}-\frac{1}{p}\right)}} w\left(B\left(y, 2^{k+1} r\right)\right)^{\frac{1}{\alpha}-\frac{1}{q}-\frac{1}{p}}\left\|f \chi_{B\left(y, 2^{k+1} r\right)}\right\|_{q_{w}}\right) \\
& +w(B(y, 2 r))^{\frac{1}{\alpha}-\frac{1}{q}-\frac{1}{p}}\left\|f \chi_{B(y, 2 r)}\right\|_{q_{w}}
\end{aligned}
$$


for all $y \in \mathbb{R}^{n}$. Therefore the $L^{p}$-norm of both sides of (4.28), gives

$$
r\left\|\left[b, S_{\gamma}\right](f)\right\|_{q_{w}, p, \alpha} \lesssim\left(1+\|b\|_{B M O}\right)\|f\|_{q_{w}, p, \alpha}
$$

for all $r>0$, since the series $\sum_{k=1}^{\infty} \frac{k+3}{2^{\frac{2 n k}{s^{\prime}}\left(\frac{1}{\alpha}-\frac{1}{p}\right)}}$ converges. We end the proof by taking the supremum over all $r>0$.

Proof of Theorem 3.4. It is easy to see that

$$
\left[b, g_{\lambda, \gamma}^{*}\right](f)^{2}(x) \lesssim \sum_{j=0}^{\infty} 2^{-j \lambda n}\left[b, S_{\gamma, 2^{j}}\right](f)^{2}(x)
$$

for all $x \in \mathbb{R}^{n}$. So, for all balls $B=B(y, r)$ we have

$$
\left\|\left[b, g_{\lambda, \gamma}^{*}\right](f) \chi_{B}\right\|_{q_{w}} \lesssim \sum_{j=0}^{\infty} 2^{-\frac{j \lambda n}{2}}\left\|\left[b, S_{\gamma, 2^{j}}\right](f) \chi_{B}\right\|_{q_{w}} .
$$

Using the arguments as in the proof of Theorems 3.3 and 3.1 and taking into consideration (4.16) we end the proof.

\section{References}

[1] J. Feuto, Norms inequalities in some subspaces of Morrey space, Preprint.

[2] F. Chiarenza and M. Frasca, Morrey spaces and Hardy-Littlewood maximal function, Rend. Mat. Appl. (7) 7 (1987), no. 3-4, 273-279.

[3] R. Coifman and C. Fefferman, Weighted norm inequalities for maximal functions and singular integrals, Studia Math. 51 (1974), 241-250.

[4] D. Fan, Sh. Lu, and D. Yang, Regularity in Morrey spaces of strong solutions to nondivergence elliptic equations with VMO coefficients, Georgian Math. J. 5 (1998), no. 5, 425-440.

[5] J. Feuto, I. Fofana, and K. Koua, Espaces de fonctions á moyenne fractionnaire intégrable sur les groupes localement compacts, Afrika Mat. (3) 15 (2003), 73-91.

[6] - Weighted norms inequalities for a maximal operator in some subspace of amalgams, Canad. Math. Bull. 53 (2010), no. 2, 263-277.

[7] - Integrable fractional mean functions on spaces of homogeneous type, Afr. Diaspora J. Math. 9 (2010), no. 1, 8-30.

[8] I. Fofana, Étude d'une classe d'espaces de fonctions contenant les espaces de Lorentz, Afrika Mat. (2) 1 (1988), no. 1, 29-50.

[9] J. J. F. Fournier and J. Stewart, Amalgams of $L^{p}$ and $L^{q}$, Bull. Amer. Math. Soc. 13 (1985), no. 1, 1-21.

[10] L. Grafakos, Modern Fourier Analysis, Second Edition, Springer, 2009.

[11] F. Holland, Harmonic Analysis on amalgams of $L^{p}$ and $\ell^{q}$, J. London Math. Soc. (2) 10 (1975), 295-305.

[12] F. John and L. Nirenberg, On functions of bounded mean oscillation, Comm. Pure Appl. Math. 14 (1961), 415-426.

[13] C. B. Morrey, On the solutions of quasi-linear elliptic partial differential equations, Trans. Amer. Math. Soc. 43 (1938), no. 1, 126-166.

[14] B. Muckenhoupt, Weighted norm inequalities for the Hardy maximal function, Trans. Amer. Math. Soc. 165 (1972), 207-226.

[15] Y. Komori and S. Shirai, Weighted Morrey spaces and a singular integral operator, Math. Nachr. 282 (2009), no. 2, 219-231; DOI 10.1002/mana.200610733. 
[16] H. Wang, Intrinsic square functions on the weighted Morrey spaces, J. Math. Anal. Appl. 396 (2012), no. 1, 302-314.

[17] M. Wilson, The intrinsic square function, Rev. Mat. Iberoam. 23 (2007), no. 3, 771-791.

[18] — Weighted Littlewood-Paley Theory and Exponential-Square Integrability, Lecture Notes in Math, Vol 1924, Springer-Verlag, 2007.

Laboratoire de Mathématiques Fondamentales

UFR MathÉmatiques et Informatique

Université de CoCOdy

22 B.P. 1194 Abidjan 22. Côte D'IVoire

E-mail address: justfeuto@yahoo.fr 